\title{
SOL, NUTRITION ET PRODUCTION LIGNEUSE
}

\author{
F. LE TACON \\ Station de Recherches sur les Sols forestiers et la Fertilisation, \\ Centre national de Recherches forestières, I. N. R. A., \\ Champenoux 54370 Einville
}

\section{RÉSUMÉ}

Depuis longtemps, les forestiers ont cherché à déterminer dans quelle mesure le sol pouvait avoir une influence sur le comportement des peuplements.

Les premières tentatives ont été effectuées de manière indirecte en utilisant les indications de la flore herbacée. Depuis, cette méthode s'est considérablement améliorée. Parallèlement, la recherche des liaisons directes entre la production et les caractéristiques du sol, sans passer par l'intermédiaire de la flore, s'est développée à partir de 1920 et surtout à partir de 1940.

Deux méthodes sont utilisées :

- la premiére consiste à mettre en évidence ces liaisons sol-production, mais sans rechercher d'explications causales : c'est la méthode comparative ou par enquête.

- la seconde consiste, au contraire, à expliquer l'action des facteurs du sol sur l'arbre à l'aide d'expérimentations, souvent à caractères écophysiologiques, permettant de contrôler un ou plusieurs facteurs.

La méthode comparative consiste à choisir un ensemble de stations permettant de couvrir toute la gamme de sols d'une région climatiquement homogène. En chacune des stations, deux catégories de mesure sont effectuées :

- des mesures de production,

- des mesures caractérisant le sol, soit directes, soit indirectes (analyses foliaires).

Le problème consiste à rechercher les liaisons existant entre ces deux catégories de mesures. Trois moyens sont utilisés :

- des méthodes simples basées sur l'analyse de la variance à un facteur contrôlé,

- des méthodes basées sur la matrice des coefficients de corrélation (analyses statistiques muitivariates),

- des méthodes basées sur des modèles non linéaires.

Ces deux dernières méthodes nécessitent l'utilisation de moyens de calculs puissants (ordinateur).

Cette méthode comparative a actuellement atteint une grande efficacité, proche de la limite de ses possibilités, bien que des améliorations soient encore possibles.

Si cette méthode comparative était utilisée de manière systématique, elle pourrait rendre à son stade actuel d'élaboration des services considérables pour la prévision de la production et la rationalisation du choix de l'essence.

La méthode expérimentale peut se concevoir d'une part par modification des caractéristiques du sol, d'autre part par la recherche des mécanismes d'action des facteurs du sol sur l'arbre (écophysiologie). 
Cette voie expérimentale permet de déterminer les véritables liaisons de cause à effet entre le sol et le végétal et donc d'intervenir par modification de certaines caractéristiques du milieu. Le développement de la fertilisation forestière est une conséquence de ce type de recherche.

L'approche écophysiologique permettra probablement de faire des progrès considérables dans la connaissance des mécanismes d'action du sol. Mais, les résultats pratiques que l'on peut en attendre sont aléatoires, et certainement pas envisageables à court terme. Ce type de recherche prendra cependant le relais des autres méthodes lorsque toutes les possibilités de ces dernières auront été épuisées.

\section{1. - INTRODUCTION}

A l'échelle du globe, ce sont les facteurs climatiques qui expliquent la plus grande part des variations de production. A l'échelle régionale au contraire, ce sont les caractéristiques de la station et souvent du sol qui déterminent ces variations.

Rappelons qu'en un point donné, la production maximale possible est déterminée par les paramètres climatiques et le potentiel du matériel végétal utilisé. Suivant la valeur des différentes caractéristiques du sol, le niveau de production réel variera entre 0 et cette production maximale possible.

Il est donc du plus haut intérêt pour le forestier de savoir, pour diverses essences et dans des conditions climatiques données, quelle sera, en fonction des caractéristiques du sol, la production qu'il pourra espérer obtenir. Il pourra ainsi choisir l'essence qui assurera la production maximale, dans la mesure évidemment oủ seul le critère de production entre en ligne de compte.

D'autre part, connaissant les facteurs limitant la production par excès ou par défaut, il pourra les corriger si les contraintes économiques le permettent.

Depuis longtemps, les forestiers ont cherché à déterminer dans quelle mesure le sol pouvait avoir une influence sur le comportement des peuplements. Les premières tentatives ont été effectuées de manière indirecte en utilisant les indications de la flore herbacéc. Depuis, cette méthode basée sur la flore s'est considérablement améliorée (voir exposé n 6). Parallèlement, la recherche des liaisons directes entre la production et les facteurs du sol, sans passer par l'intermédiaire de la flore, s'est développée à partir de 1920 et surtout à partir de 1940 , essentiellement d'ailleurs aux États-Unis.

Deux méthodes sont utilisées : la première consiste à mettre en évidence ces liaisons sol-production, mais sans rechercher d'explications causales; c'est la méthode comparative ou par enquête.

La seconde consiste au contraire à expliquer l'action des facteurs du sol sur l'arbre au moyen d'expérimentations à caractères écophysiologiques permettant de contrôler un ou plusieurs facteurs.

Depuis environ 20 ans, ces deux méthodes d'investigation ont vu leur efficacité augmenter de manière spectaculaire. L'ordinateur est devenu l'outil de travail favori de l'école comparative. D'autre part, l'amélioration de l'équipement scientifique pour les investigations à caractères écophysiologiques ne semble pas avoir de limites. D'ailleurs, depuis une époque très récente, certains physiologistes commencent à utiliser l'ordinateur pour simuler certains 
processus à partir de résultats obtenus expérimentalement. Nous allons maintenant entrer dans le détail de ces deux méthodes.

\section{II. - MÉTHODE COMPARATIVE OU PAR ENQUÊTE}

Nous allons décrire rapidement le principe de cette méthode. Si nous faisons abstraction du potentiel génétique et des techniques culturales peu employées jusqu'à ce jour en matière forestière, sauf exception par exemple pour le Pcuplier et le Pin maritime en France, la production d'une essence ne dépend plus que des caractéristiques climatiques et pédologiques.

Dans la mesure du possible, il est préférable de dissocier ces deux catégories de paramètres. Il est parfois possible d'y arriver si l'on peut délimiter une zone climatiquement peu variable. Nous nous attacherons done à installer des points d'étude ( 50 à 100 placettes ou plus) de façon à couvrir toute la gamme des stations, c'est-à-dire toutes les variations de sol et de microclimat.

En plaine, un tel échantillonnage ne pose pas trop de problèmes. En région montagneuse, par contre, les conditions climatiques et microclimatiques prennent un rôle prépondérant et ont une grande variation. Il devient alors difficile de déterminer la part qui revient au sol, d'autant plus que le climat détermine lui-même en partie le type de sol et par conséquent ses caractéristiques.

Mais supposons néanmoins que les problèmes d'échantillonnage ont pu être résolus. En chacun des points ou des placettes de l'échantillon, nous pouvons effectuer deux catégories de mesure :

- des mesures de production, au sol.

- des mesures caractérisant la station et qui, dans le cadre de cet exposé, se limitent

Lorsque nous sommes en possession de ces deux catégories de mesures, le problème est de rechercher les liaisons pouvant exister entre elles.

Nous ne parlerons pas des mesures de production dont il a été question par ailleurs, mais nous allons nous apesantir un peu sur la mesure des caractéristiques du sol.

\section{1 - Mesures des caractéristiques du sol}

Le sol peut en premier lieu être caractérisé par des notions synthétiques telles que le type génétique, le type d'humus ou le type de roche-mère. Mais il est évidemment nécessaire d'aller plus loin que ces notions globales et de mesurer un certain nombre de caractéristiques physiques et chimiques. Ces paramètres relatifs au sol peuvent être appréciés soit directement, soit, pour certains, indirectement par l'intermédiaire du végétal lui-même, pour lequel il est possible d'effectuer certains diagnostics de nutrition. Voyons tout d'abord le problème des mesures directes.

\subsection{Mesures directes.}

Il est inutile d'insister sur la difficulté des déterminations des propriétés du sol en général. Nous possédons diverses techniques permettant d'apprécier avec plus ou moins d'appro- 
ximation certaines de ses caractéristiques ou certains de ses états. Ces techniques continuent de progresser et de s'affiner; mais à partir d'un certain degré de complexité, elles ne peuvent plus être utilisćes pour des études nécessitant un trẻs grand nombre de déterminations. Il est donc nécessaire de rester à un certain niveau de simplicité et par conséquent d'approximation.

D'autre part les caractéristiques physico-chimiques d'un sol varient avec la profondeur. Il est donc indispensable de les mesurer à différents niveaux, d'autant plus que l'arbre prospecte le sol sur une grande profondeur, de l'ordre du mètre et parfois beaucoup plus.

Une première solution consiste à effectuer les prélèvements à profondeur constante. Néanmoins, les horizons étant variables d'un sol à l'autre aussi bien par leur nature que leur nombre ou leur épaisseur, cette manière de procéder pose quelques difficultés.

Il est aussi possible d'effectuer des prélèvements dans tous les horizons c'est-à-dire par niveau homogène. On exprime alors les résultats en volume de sol par tranches successives d'égale épaisseur, en tenant compte de la densité apparente de l'épaisseur et de la teneur en cailloux de chaque horizon. Nous pouvons ainsi obtenir une série de chiffres comparables pour tous les profils. II reste le cas des variables pour lesquelles on ne peut effectuer de sommation, comme par exemple le $\mathrm{pH}$ ou la densité apparente. Pour ces facteurs il existe en général un gradient de variation en fonction de la profondeur. Nous pouvons alors essayer de mesurer la profondeur à laquelle ce facteur prend une valeur déterminée.

Quel que soit le procédé adopté, nous sommes en présence, soit de variables mesurées à profondeur constante, soit de variables exprimées par tranche de sol d'épaisseur constante, soit de variables prenant des valeurs constantes à profondeur variable. Nous pouvons garder tous ces chiffres pour les interprétations ultérieures ou essayer de réduire le nombre de variables en cherchant les corrélations existant entre elles. Si les valeurs que prennent un facteur en fonction de la profondeur sont très corrélées, il sera possible de réduire ce facteur à une seule valeur. Si il n'y a pas de corrélation ou si les corrélations sont lâches, la réduction n'est pas envisageable. (MILIER, Le TACON, 1971.)

Dans certains cas privilégiés, il sera possible de ne prendre en compte que les caractéristiques des horizons supérieurs, c'est-â-dire de l'humus qui intégre, en forêt naturelle, certaines propriétés de l'ensemble du profil.

D'autre part, il se pose très rapidement un problème de choix. Nous pouvons mesurer un très grand nombre de paramètres, mais il est hors de question de les mesurer tous. II faut donc au préalable ne retenir que les paramètres qui semblent avoir le plus de chance d'influer sur le comportement des peuplements étudiés. C'est un choix difficile, lourd de conséquences. Mais nos connaissances sont actuellement suffisantes, au moins pour les sols français, pour nous permettre d'effectuer ce choix sans de trop grands risques d'erreurs.

D'autres difficultés attendent les écologistes s'intéressant aux liaisons existant entre le sol et le végétal par la méthode comparative. II s'agit essentiellement des facteurs dépendant des conditions climatiques annuelles, c'est-à-dire principalement tout ce qui concerne I'alimentation en eau et la nutrition azotée.

Voyons tout d'abord le problème de l'eau. Nous pouvons avoir assez facilement accès à la capacité maximale de rétention en eau utile. Mais cette notion est bien insuffisante. En effet, à capacité de rétention en eau utile équivalente, deux sols peuvent présenter une dispo- 
nibilité en eau très différente suivant, par exemple, la valeur des apports latéraux ou de profondeur. De même la présence d'une nappe, quelle soit temporaire ou permanente, vient singulièrement compliquer le problème.

Il est possible d'établir des profils hydriques pendant une année ou plusieurs années consécutives comme le fait Lévy pour l'étude qu'il a entreprise, et qui concerne l'épicéa sur les sols hydromorphes du Nord-Est de la France. Mais en raison de la multiplicité des observations nécessaires à l'établissement de tels profils, il est impossible de les effectuer sur un très grand nombre de points.

De plus, le peuplement lui-même intervient dans le bilan hydrique, comme le montrent par exemple les travaux de Aussenac, en interceptant et en évapotranspirant une plus ou moins grande quantité d'eau suivant son âge, sa nature et le traitement qu'on lui applique. II est donc très difficile de trouver une solution satisfaisante à ce problème de l'alimentation en eau.

Une solution peut, semble-t-il, être apportée par la flore herbacée dans certaines forêts naturelles. BECKER, en forêt de Charmes, sur sols hydromorphes, dans l'Est de la France, a montré de façon très convaincante que la présence de certaines espèces herbacées étaient exactement en rapport avec le régime hydrique des sols.

Le problème de la disponibilité en azote des sols est tout aussi ardu. Cette disponibilité varie suivant les années et les périodes de l'année. A un instant donné, la quantité d'azote minéral présent dans un horizon dépend d'un grand nombre de phènomènes : minéralisation brute, réorganisation, absorption par les racines, pertes par drainage ou échanges gazeux, rétrogradation, fixation d'azote atmosphérique, apport par les eaux de pluie et par les caux de percolation à travers la masse foliaire, etc.

La meilleure méthode est de doser périodiquement l'azote minéral dans le sol et dans les eaux de drainage d'un lysimètre, comme l'a effectué récemment BonnEAu dans les Vosges. Cette méthode n'est applicable malheureusement qu'à un trẻs petit nombre de stations.

Nous pouvons l'alléger en dosant périodiquement in situ l'azote minéral existant dans le sol, soit sans prendre de précautions particulières, soit en protégeant les horizons contre les apports extérieurs ou les pertes par drainage ou par absorption par les racines (LEMÉE, 1967).

Un procédé beaucoup plus commode consiste à déterminer la production d'azote minéral d'un échantillon à l'ćtuve, dans des conditions bien définies de température et d'humidité. Malheureusement, la production d'azote ainsi obtenu n'est pas toujours en rapport étroit avec la production réelle d'azote d'un sol en place, comme l'a démontré LeMÉE (1967). Néanmoins, récemment, nous avons obtenu par ce procédé des résultats plus convaincants que ceux auxquels nous pouvions nous attendre (LE TACON 1972).

Heureusement les possibilités d'alimentation azotée peuvent être appréciées indirectement, comme d'ailleurs celles des autres éléments minéraux. Nous en arrivons ainsi aux méthodes indirectes d'appréciation des caractéristiques du sol.

\subsection{Méthodes indirectes (diagnostic de mutrition).}

Devant la complexité des méthodes de caractérisation du sol, ${ }^{5}$ les agronomes ont très tôt essayé de déterminer les possibilités d'alimentation minérale en utilisant la plante elle- 
même, soit sa totalité, soit certains organes particuliers. L'analyse minérale totale des fcuilles est la plus utilisée, aussi bien en agronomie qu'en matière forestière. Signalons que cette technique a dû être appliquée pour la première fois à des arbres forestiers en 1873 par FLICHE et Grandeau, qui ont travaillé sur Pin maritime et Pin noir d'Autriche.

La teneur en un élément minéral présent dans les feuilles fournit un indice de la disponibilité de cet élément dans le sol. C'est un indice particuliẻrement facile à obtenir; l'analyse minérale totale des tissus est en effet très rapide et ne pose pas de difficultés particulières. C'est de plus une mesure facile à manier qui fournit un seul chiffre pour caractériser les possibilités d'alimentation en un élément pour l'ensemble d'un profil. Ceci facilite considérablement le travail d'interprétation ultérieur. II n'en reste pas moins que, malgré tous ces avantages, plusieurs problèmes demeurent. Ils sont bien connus depuis longtemps, aussi allons-nous énumérer les plus importants très rapidement.

- Il n'y a pas souvent parallélisme entre la concentration d'un élément dans les tissus, sa concentration dans le sol et la croissance. Il suffit de se reporter à l'équation de MitsCherLich dont nous aurons l'occasion de reparler.

- Il existe très fréquemment des phénomènes d'antagonisme ou de synergie entre éléments au niveau de l'absorption racinaire.

- La concentration en un élément dans les feuilles subit des variations annuelles importantes et parfois même des variations journalières. De plus, l'allure de ces courbes de variation est trẻs différente suivant l'élément considéré.

- Il se produit souvent des phénomẻnes de concentration ou de dilution des éléments minéraux suivant la quantité de matière sèche produite. Lorsque la croissance est faible, par exemple en raison des conditions climatiques défavorables, la demande en éléments minéraux est faible. Dans ces conditions, même si le sol est pauvre, la teneur en éléments minéraux des feuilles est plus ou moins normale. Lorsque les conditions climatiques deviennent plus favorables, par exemple lorsque l'altitude diminue, la masse foliaire produite est beaucoup plus importante. La demande en éléments minéraux est donc beaucoup plus forte. Les éléments se diluent dans la masse foliaire, ce qui entraine une diminution de leur concentration dans les tissus foliaires. Cette baisse de concentration n'a évidemment rien à voir avec une diminution de fertilité.

Dans l'Ardèche, avec l'Épicéa, nous avons été confronté avec ce problème connu depuis longtemps (Le TACON, Oswald, Tomassone, 1970) comme d'ailleurs Chichery (1971) plus récemment avec le Sapin pectiné dans les Vosges.

Enfin, si la teneur en éléments minéraux d'un organe dépend de la fertilité du sol, elle dépend aussi de beaucoup d'autres phénomènes tels que les migrations et les transferts vers d'autres organes.

L'analyse foliaire est done un moyen commode d'apprécier la disponibilité d'un sol en éléments minéraux, mais elle n'est en fait qu'un miroir déformant.

Il serait donc du plus haut intérêt de pouvoir éliminer ces déformations, ce qui nous conduit à dire quelques mots d'autres techniques de diagnostic telles que l'analyse racinaire et l'analyse de la sève brute.

Très récemment BLum (1971) a tenté d'utiliser des racines pour établir un diagnostic de nutrition. Il s'avère que pour l'Épicéa, il existe d'excellentes corrélations entre la teneur en 
éléments minéraux des racines de diamètre inférieur à $2 \mathrm{~mm}$ et prélevées dans les 10 premiers centimètres du sol, et les réserves du sol en éléments minéraux. Cette technique pourrait donc fournir un moyen intéressant de diagnostic.

D'autres auteurs ont pensé utiliser la sève brute et déterminer ainsi les éléments absorbés directement par l'arbre. Malheureusement cette méthode pose des problèmes techniques encore non résolus. BOLLARD, dès 1953, a mis au point une technique qui consiste à sectionner un rameau de la cime et à extraire la sève brute par pression. On obtient en moyenne $1 \mathrm{ml}$ de sève pour $100 \mathrm{~g}$ de branche sèche. Cette méthode n'est donc guère applicable à un grand nombre d'arbres.

L'idéal serait d'avoir accès à la sève brute du tronc. Mais pour l'instant personne semble-t-il n'est arrivé à des résultats satisfaisants.

\section{2. - Moyens permettant de déterminer linfluence des caractéristiques du sol sur la production}

Nous sommes maintenant en présence de deux séries de variables : la production qu'il s'agit "d'expliquer " et les caractéristiques du sol ou variables "explicatives ", qu'elles soient mesurées directement ou indirectement par l'intermédiaire d'un diagnostic de nutrition.

II faut immédiatement s'apesantir sur le sens du terme « expliquer » utilisé ici et qu'il faut mettre entre guillemets. En effet, qui dit explication, dit connaissance des liaisons de cause à effet. Or, à ce stade, c'est-à-dire au stade comparatif, nous constatons seulement que certains facteurs varient soit dans le même sens que la production, soit en sens inverse. Il est donc possible de tirer parti de ces constatations pour prévoir que si certains facteurs de station ou de sol atteignent une certaine valeur, la production atteindra une valeur correspondante. Mais cela ne veut pas dire que ce sont ces facteurs qui sont réellement responsables des variations de production.

Nous conserverons néanmoins le terme d'explication, mais en lui donnant le sens restrictif d'explication apparente.

II faut maintenant faire la distinction entre les peuplements naturels et les peuplements artificiels. Dans le cas des peuplements artificiels, nous pouvons espérer que la variabilité génétique est répartie de manière aléatoire, c'est-à-dire de la même façon suivant les sols ou les stations. Suivant l'importance respective de la variabilité du milieu et de la variabilité génétique, l'explication des variations de production par les facteurs du milieu sera plus ou moins bonne. Les fourchettes de prévision seront donc plus ou moins étroites, mais toujours exactes dans leur application.

Dans le cas des peuplements naturels, le problème est très différent. Nous pouvons en effet être certain que sur un tel matériel sauvage, les conditions de milieu ont joué et qu'il y à eu adaptation aux conditions de station, et donc au sol. Comme dans le cas des peuplements artificiels, il sera possible apparemment d'expliquer la production au moyen des variables du milieu. Il est d'ailleurs prouvé que le pourcentage d'explication apparent est bien meilleur en général pour les forêts naturelles que pour les forêts artificielles. 
Mais que recouvre en réalité ce pourcentage d'explication? II recouvre, d'une part l'explication apparente propre du milieu, équivalente à celle des peuplements artificiels, et d'autre part l'explication génétique imputable ả l'adaptation au milieu.

Il en résulte que les procédés de prévision risquent de se révéler en partie inutilisables si, pour un sol donné, on remplace la provenance autochtone par une provenance étrangère à la station.

Ces réserves étant faites, voyons maintenant comment il est possible de relier la production aux caractéristiques du sol. Trois types de méthodes peuvent être utilisés :

- des méthodes simples basées sur l'analyse de la variance à un facteur contrôlé,

- des méthodes basées sur la matrice des coefficients de corrélation,

- des méthodes basées sur des modèles non linéaires.

\subsection{Méthodes simples basées sur l'analyse de la variance à un facteur contrölé.}

Le facteur contrôlé qui sert à expliquer les différences de production entre peuplements est soit qualitatif et synthétique, soit quantitatif. Dans le premier cas, ce facteur pourra être le type de sol ou le type d'humus ou le type de roche-mẻre; dans le second, ce pourra être n'importe quel facteur mesuré au cours de l'enquête ou une combinaison de ces facteurs.

Ces facteurs qualitatifs ou quantitatifs peuvent fournir une discrimination excellente vis-à-vis de la production, et donc fournir d'excellents moyens de prévision de la production. Nous allons prendre deux exemples qui vont nous permettre d'illustrer ce que l'on peut attendre de cette méthode. Ils ont trait tous les deux à l'Épicéa commun, à peu près sur le même type de roche-mère, c'est-ả-dire sur roche-mère calcaire, mais de part et d'autre du massif vosgien et de la Forêt noire. L'un en effet est pris en Allemagne dans l'Alpe souabe (Werner, 1962), l'autre sur les plateaux calcaires du Nord-Est de la France (Decourt, LE TACON, 1970).

Voyons tout d'abord I'exemple allemand, La figure I représente la variation de la production moyenne de l'Épicéa à 100 ans $\mathrm{en} \mathrm{m}^{3} /$ ha/an en fonction des types de roche-mère et des types de sol correspondant. Le facteur contrôlé est done ici un facteur synthétique. Sans entrer dans le détail, nous voyons que la production de l'Épicéa est minimale sur les sols carbonatés à pH élevé et à réserve en eau faible. Elle augmente au fur et à mesure que les différents sols voient leur profondeur augmenter et leur pH diminuer, et devient maximale sur les sols développés sur terra fusca et limons.

Sur le graphique $n^{\circ} 2$, WERNER a représenté la variation de la nutrition en azote et en phosphore de l'épicéa en fonction de ces mêmes types de roches-mères et de types de sol. Nous voyons que la nutrition azotée et la nutrition en phosphore varient dans le même sens que la production. Cela est particulièrement net pour la nutrition azotée, qui est minimale (teneur en $\mathrm{N}$ voisin de 1 p. 100), comme la production, sur les sols à $\mathrm{pH}$ élevé, et optimale (teneur en $\mathrm{N}$ voisine de 1,6 p. 100) sur les sols acides développés sur limons et terra fusca.

Voyons maintenant l'exemple de l'Épicéa sur les plateaux calcaires de l'Est de la France (Decourt, Le TACON, 1970).

Nous n'entrerons pas non plus dans le détail de cette étude qui a été publiée par ailleurs et à laquelle on pourra se reporter. 


\begin{tabular}{|c|c|}
\hline Type de station & $\begin{array}{l}\text { Production moyenne à } 100 \text { ans } \\
\text { depuis l'origine en } \mathrm{m}^{3} / \mathrm{ha} / \mathrm{an} \\
2,4,6,10,10,12,16\end{array}$ \\
\hline $\begin{array}{l}1 \text { Limons provenont de t'altáration } \\
\text { de dolomies (1 1̇egénérotion) }\end{array}$ & mal \\
\hline $\begin{array}{l}2 \text { Sols peu évolués sur mernes } \\
\text { (1êre générotion) }\end{array}$ & \\
\hline $\begin{array}{l}3 \text { Sols superficiels sur colcaire } \\
\text { tertioire - a) } 1 \text { ė̃e génération } \\
\text { b) après plus d'une génération }\end{array}$ & . \\
\hline $\begin{array}{l}4 \text { Sols moyennement superficiels } \\
\text { sur calcairo tertioire }\end{array}$ & \\
\hline $\begin{array}{l}\text { Matériel limeneux peu épais issu } \\
\text { de l'oltération du caleaire }\end{array}$ & \\
\hline $\begin{array}{l}6 \text { Matériel Limoneux moyennement } \\
\text { épois issu de l'oltération du calc, }\end{array}$ & \\
\hline 7 Limons hétérogènes & \\
\hline 8 Limons purs & 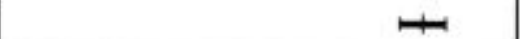 \\
\hline
\end{tabular}

FiG. 1. - Prodaction moyenne de Tépicéa à 100 ans depwis Torigine sur différentes stations du centre de V'Alpe Somabe d'après Werner 1962

FIG. 1. - Mean annual volume increments (from Oto 100 years) in relation to soil characteristics in a Schwäbischen Alh w (Werner 1962)

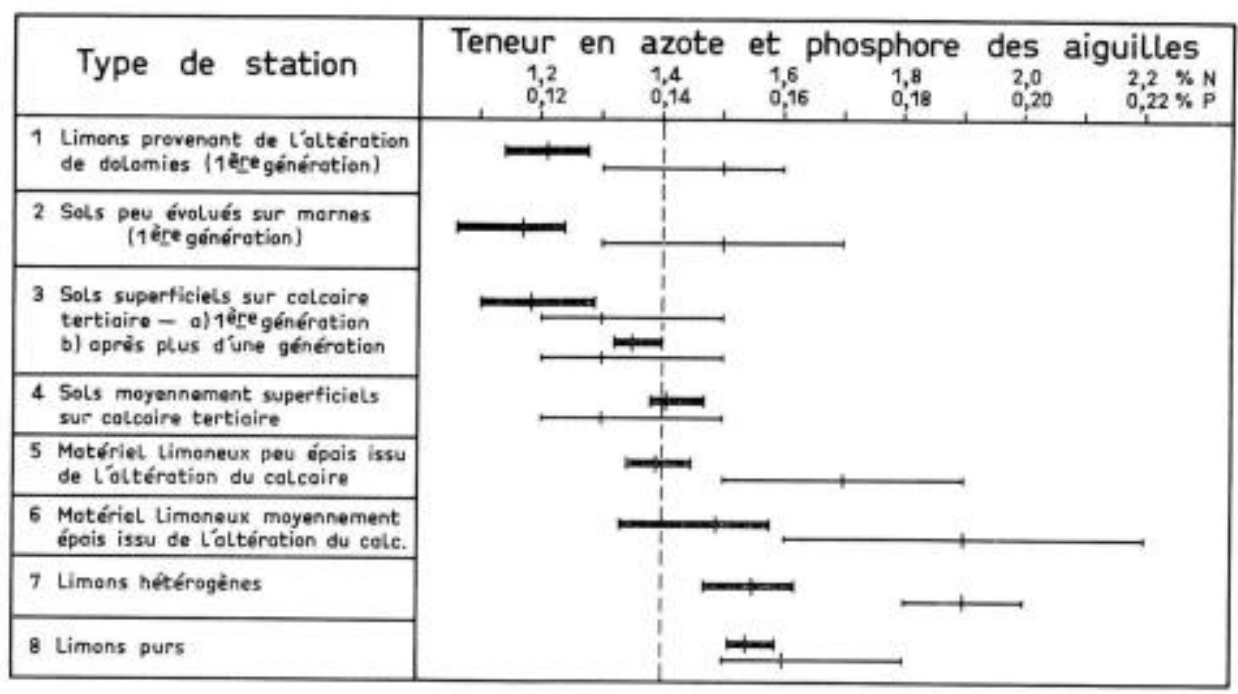

FIG. 2, - Variation de la teneur en azote ef en phosphore des aiguilles d'épicéa en fonction des differents types de stations du centre de I'Alpe Sonabe, (Werner 1962)

FIG. 2. - Variation of nitrogen and phosphorus content of spruce necdles in relation to soil characteristics in \& Schwibischen Alb * (Werner 1962) 
Sur un graphique, non représenté ici, nous avons figuré la variation de la hauteur dominante de l'épicéa à 50 ans en fonction d'ensembles de sols, définis par des critères mesurables et non par des facteurs synthétiques comme dans l'exemple précédent.

Les résultats sont très voisins de ceux de WERNER. La hauteur dominante de I'Épicéa est minimale sur les sols les plus carbonatés et maximale lorsque la profondeur de décarbonatation est la plus grande. Une exception cependant est constituée par les sols carbonatés à alimentation en eau optimale (apports latéraux) et sur lesquels l'Épicéa peut avoir une production très élevée.

On conçoit qu'à partir de tels graphiques, il soit possible de donner, soit des fourchettes de prévision de production en fonction du type de sol, soit des critères permettant de déterminer des seuils de production minimale. Par exemple pour l'Épicéa, dans le Nord-Est de la France, nous avons pu donner des critẻres très simples permettant de prévoir que la production sera au moins égale à $10 \mathrm{~m}^{3} / \mathrm{ha} / \mathrm{an}$ à $50 \mathrm{ans}$, ou à $8 \mathrm{~m}^{3}$ ou à $6 \mathrm{~m}^{3}$ ou à $3 \mathrm{~m}^{3} / \mathrm{ha} / \mathrm{an}$ à 50 ans.

C'est donc une méthode excellente qui doit toujours être utilisée quand cela est possible. Malheureusement ce n'est pas toujours aussi simple, car les facteurs qui agissent sur la production ne sont pas toujours évidents. En général, il n'y a pas un facteur qui prime sur les autres, comme dans les exemples que nous avons vus, mais un grand nombre de facteurs, qui agissent chacun pour une plus ou moins grande part et qui, souvent, sont corrélés entre eux, se compensent ou interfèrent.

Dans ces conditions seules des analyses statistiques plus élaborées permettent d'arriver à un résultat.

\subsection{Méthodes bastes sur la matrice des coefficients de corrélation.}

Nous arrivons donc au deuxième type de méthode basée sur la matrice des coefficients de corrélation qui mesurent le degré de liaison linéaire entre deux caractères.

A partir de ces coefficients de corrélation, nous pouvons calculer, par divers procédés plus ou moins élaborés, des équations permettant de déterminer la production en fonction de variables du milieu ou de combinaisons de variables. On aboutit ainsi à des équations prédictives dont nous allons donner trois exemples.

Dans le premier exemple, il s'agit toujours de l'Épicéa dans l'Alpe souabe (Werner 1962).

$$
\begin{aligned}
& d \mathrm{GZ}_{100}=-24,8+21,2 \mathrm{~N}+31,2 \mathrm{P}, \\
& d \mathrm{GZ}_{100}=\text { production moyenne/ha/an à } 100 \text { ans, } \\
& \mathrm{N}=\text { teneur en } \mathrm{N} \text { des aiguilles en p. } 100, \\
& \mathrm{P}=\text { teneur en } \mathrm{P} \text { des aiguilles en p. } 100, \\
& \text { Pourcentage d'explication }=80 \text { p. } 100 .
\end{aligned}
$$

On ne peut pas vraiment parler d'équation prédictive dans ce cas, puisque la production est calculée à partir de la teneur en éléments minéraux des aiguilles. Mais cette équation est intéressante, car elle montre que les seules teneurs en azote et en phosphore des aiguilles peuvent " expliquer $\$ 80$ p. 100 de la variation de production. 
Le deuxième exemple d'équation a trait à l'Épicéa sur les plateaux calcaires du NordEst de la France. Il s'agit cette fois d'une véritable équation de prédiction, puisque la hauteur dominante est calculée à l'aide de 6 variables du milieu.

Épicéa plateaux calcaires du Nord-Est (Militer, Le TACon, 1971).

$\mathrm{H}_{\mathrm{D}} 50=31,96-0,010\left(\mathrm{X}_{1}\right)+0,015 \mathrm{X}_{2}+0,008 \mathrm{X}_{3}+0,002 \mathrm{X}_{4}-1,86 \mathrm{X}_{5}-0,05 \mathrm{x} \mathrm{X}_{6}$. Pourcentage d'explication $=50$ p. 100.

$\mathrm{H}_{\mathrm{D}} \mathrm{SO}=$ hauteur dominante à 50 ans $\mathrm{en} \mathrm{m}$.

$\mathrm{X}_{1}=$ profondeur de décarbonatation

$\mathrm{X}_{2}=$ profondeur à laquelle $\mathrm{d}^{\prime} \geqslant 1,5\left(\mathrm{~d}^{\prime}=\right.$ densité apparente).

$\mathrm{X}_{3}=$ capacité de rétention en eau utile.

$\mathrm{X}_{4}=$ phosphore assimilable en gramme par $\mathrm{m}^{2}$

$\mathrm{X}_{5}=\mathrm{pH} \mathrm{A_{1 }}$.

$\mathrm{X}_{6}=$ calcaire total en $\mathrm{A}_{1}$.

Le pourcentage d'explication est relativement faible (50 p. 100), mais il est à noter que, par l'intermédiaire de la nutrition, ce pourcentage d' " explication " est amélioré puisqu'il atteint 60 p. 100 .

Le troisième exemple que nous citerons a trait à l'Épicéa de Sitka en Grande-Bretagne (PAGe, 1970).

Epicéa de Sitka en Grande-Bretagne (PAGE, 1970).

$\mathrm{H}_{\mathrm{D}} 50=119,8-0,042 \mathrm{X}_{1}+4,28 \mathrm{X}_{2}+3,92 \mathrm{X}_{3}+2,62 \mathrm{X}_{4}-0,13 \mathrm{X}_{5}-59,83 \mathrm{X}_{6}+$ $27,04 X_{7}-$ Pourcentage d'explication $=78$ p. 100.

$\mathrm{H}_{\mathrm{D}} 50=$ hauteur dominante à 50 ans en pieds.

$\mathrm{X}_{1}=$ altitude en pieds.

$\mathrm{X}_{2}$ et $\mathrm{X}_{3}=$ positions topographiques transformées numériquement.

$\mathrm{X}_{4}=$ couleur du sol à $15 \mathrm{~cm}$ de profondeur.

$\mathrm{X}_{5}=$ humidité équivalente à $15 \mathrm{~cm}$ de profondeur.

$\mathrm{X}_{6}=$ densité apparente à $15 \mathrm{~cm}$.

$\mathrm{X}_{7}=$ densité apparente à $30 \mathrm{~cm}$.

La hauteur dominante de l'Épicéa de Sitka à 50 ans est calculée à partir de sept variables qui permettent d'obtenir un pourcentage d'explication de l'ordre de $80 \mathrm{p}$. 100 . Il est à noter que, dans ces variables du milieu, interviennent des variables de station autres que le sol (altitude et position topographique) et que les variables relatives à la position topographique peuvent être considérées comme des variables synthètiques.

A partir de ces équations de prévision, on peut facilement établir des cartes de potentialité. Dans une zone à aménager, on trace un maillage plus ou moins lâche suivant la précision que l'on veut obtenir. En chaque point, on mesure les variables intervenant dans l'équation prédictive. On peut donc calculer en chaque point une production potentielle pour une essence donnée ou plusieurs essences. Il est ensuite possible de tracer des courbes d'isopotentialité. PAGE, dans l'étude citée ici, a fourni un excellent exemple de carte de potentialité (fig. 3).

Les courbes d'isopotentialité sont exprimées en hauteur dominante à 50 ans, et la différence entre deux courbes correspond à des différences de hauteur dominante de $3 \mathrm{~m}$. 


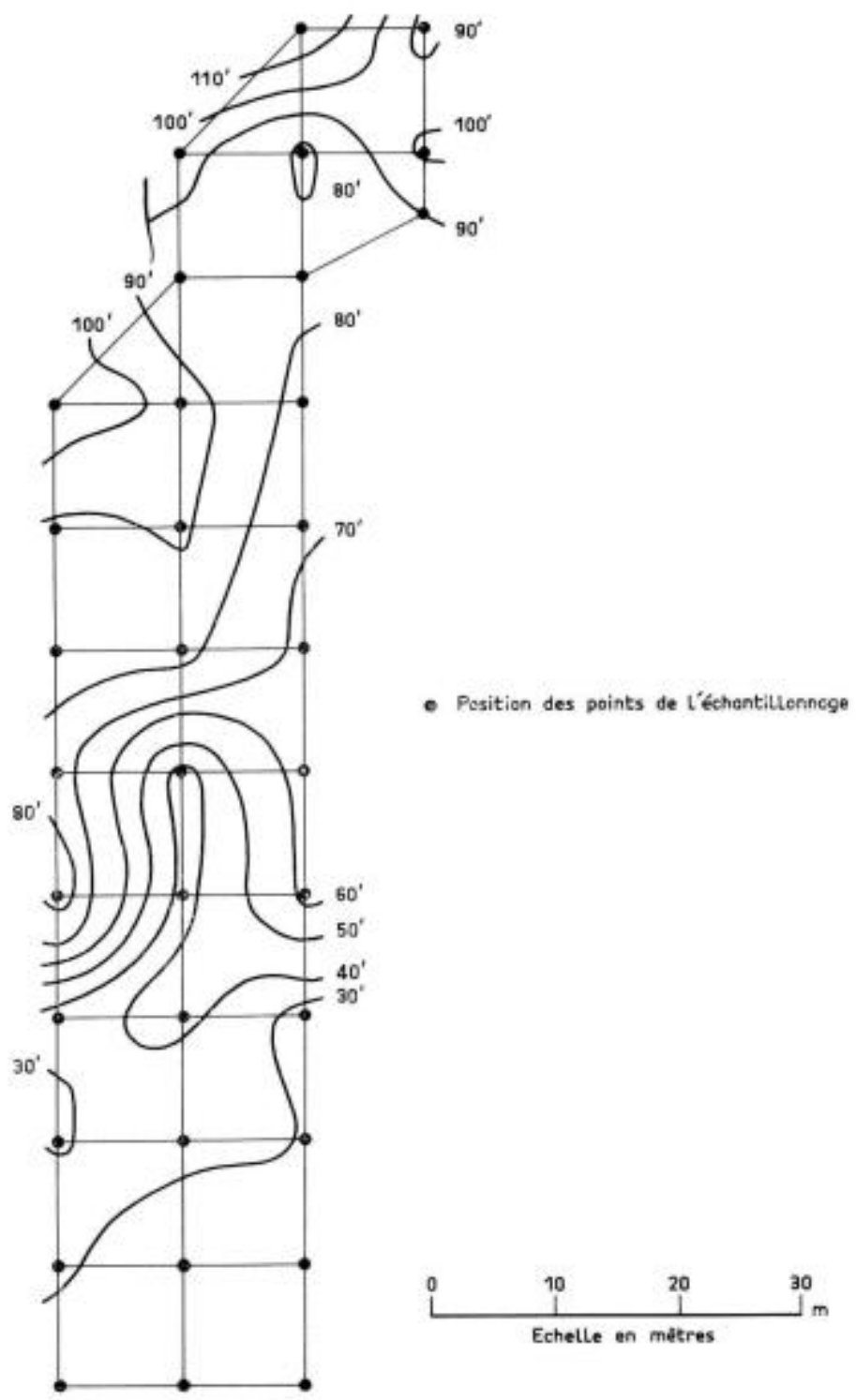

Fı. 3. - Courbes d'isopotentialité de Tépicéa de Sirka : prévixion de la hauteur dominame à 50 ans en pieds (Tintervalle entre deux courbes est de 100 pieds, soil 3,048 mètres) d'après Page, G, 1970 FiG. 3. - Preticted top height contows 50 years old Sitka spruce (Page G., 1970) 


\subsection{Inconvénients de ces méthodes.}

Si ces méthodes d'analyses multivariates basées sur la matrice des cofficients de corrélation ont un grand intérêt, comme l'ont montré ces quelques exemples, elles présentent aussi beaucoup d'inconvénients, ne serait-ce que par leur simplicité en regard de la complexité des phénomènes biologiques. Nous ne nous étendrons pas sur ces inconvénients, qui seront abondamment traités dans l'exposé $n^{\circ} 10$ (MiLlier). Nous dirons simplement quelques mots sur deux problèmes : celui de la causalité et celui de la non-linéarité des phénomènes biologiques. Le cæafficient de corrélation, nous l'avons déjà dit, mesure le degré de liaison linéaire entre deux caractères et ne préjuge en rien des liaisons de cause à effet, ce qui est très gênant pour le biologiste qui a toujours besoin de raisonner en terme de causalité. Il ne peut done le faire, en toute rigueur, à partir des résultats des analyses multivariates, que pour émettre des hypothèses. Il doit ensuite les vérifier par recoupement, et surtout par expérimentation comme nous le verrons dans un prochain paragraphe.

D'autre part, nous savons qu'en biologie les liaisons linéaires ne sont pas les plus fréquentes. Traiter tous ces problèmes de liaison station-production par l'intermédiaire du coefficient de corrélation est une approximation assez grossière, qui entraîne une perte d'information considérable.

Néanmoins cet écueil de la non-linéarité de la plupart des liaisons biologiques peut être évité grâce à l'utilisation de modèles.

\subsection{Méthodes basées sur des modèles.}

Un excellent exemple peut être fourni par le travail effectué sur Pinus radiata par Czarnowski, Humphreys et Gentle en Australie. Cet exemple est assez compliqué, car il tient compte à la fois des caractéristiques du sol et des caractéristiques climatiques. Les caractéristiques du sol prises en compte sont les suivantes : phosphore total, éléments échangeables $(\mathrm{Ca}, \mathrm{K}, \mathrm{Mg}$, et $\mathrm{Al}$ mesurés à deux profondeurs : $0-7,5 \mathrm{~cm}$ et 30 à $40 \mathrm{~cm})$, la granulométrie et la densité apparente. Pour les caractéristiques climatiques, il s'agit de la pluviosité moyenne annuelle, de la température moyenne annuelle et de l'humidité relative moyenne annuelle.

En ce qui concerne l'action des facteurs du sol, le modẻle utilisé est l'équation de MirsCherL.ISCH, qui est une exponentielle de base 10:y= A $\left(1-10^{-c x}\right)$ et qui donne la croissance en fonction de la concentration d'un élément dans le milieu. Cette équation dite " de $1^{\text {re }}$ approximation $n$ a ensuite été corrigée pour tenir compte de l'effet de toxicité par introduction d'un terme correctif $\mathrm{K}$. Les courbes ainsi obtenues avec l'équation dite de " $2^{\circ}$ approximation" suivant la valeur du terme correctif $K$ sont représentées sur la figure 4 .

Cette équation date de 1928 et est applicable à un seul facteur. Ce modéle a ensuite été amélioré par divers auteurs en permettant de combiner l'action de plusieurs facteurs (voir exposé no 10, Millier). A partir de ce modèle, et par approximations successives, Czarnowski, qui est le mathématicien de l'équipe, a calculé les termes de l'équation donnant la hauteur dominante en fonction des caractéristiques du sol.

II a ensuite calculé cette même hauteur dominante à partir des caractéristiques climatiques à l'aide d'un modèle d'équation qu'il avait déjà mis au point auparavant. Toujours 


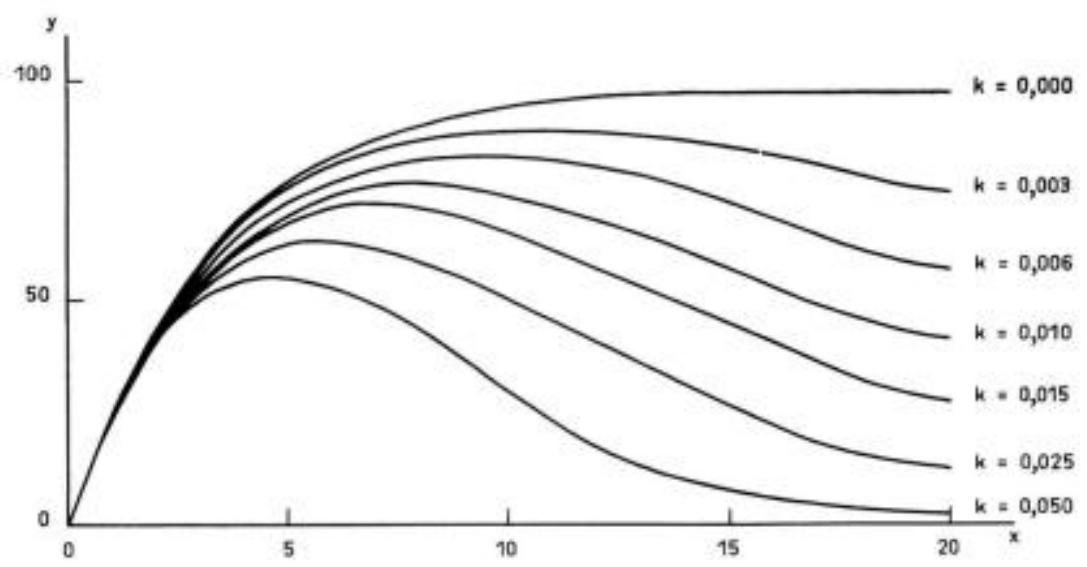

Fig. 4. - Loi de Mirscherlich de seconde approximarion y $=A\left(I-10^{-e x a}\right) \cdot 10^{-k x}$ $\left(\mathrm{y}=\mathrm{A}\left(I-10 \cdot \mathrm{ex}^{2}\right) \boldsymbol{I}^{\text {te }}\right.$ approximation)

Fic. 4. - Mitscherlich law ; second approximation y $=A\left(1-10^{-c x}\right) \cdot 10^{-k x z}$ $\left(\mathrm{y}=A\left(1-10^{-e x}\right)\right.$ first approximation $)$

par approximations successives, il a ensuite combiné les deux équations pour estimer la hauteur dominante à la fois en fonction des caractéristiques du sol et des caractéristiques climatiques. Les résultats de cette étude sont résumés par la figure 5 .

L'équation donnant $\mathrm{H}_{\mathrm{D}} 20$ en fonction du facteur du milieu est évidemment très compliquée. Nous avons en ordonnée $\mathrm{H}_{\mathrm{D}} 20$ calculée à partir de l'équation et en abscisse $\mathrm{H}_{\mathrm{D}} 20$ mesurée sur le terrain. Le résultat est assez satisfaisant puisque l'on peut prévoir ainsi la hauteur dominante et donc la production avec une erreur de $\pm 10 \%$.

La méthode comparative n'est évidemment pas la seule pour choisir rationnellement l'essence qui assurera la meilleure production sur une station donnée. La meilleure méthode, au moins en théorie, est évidemment celle des plantations comparatives d'espèces ou de provenances sur divers types de sols. Mais il n'est pas de notre propos d'aborder cet aspect de la question ici.

Choisir une essence est une chose, pouvoir intervenir en modifiant les conditions de milieu est une autre. Pour pouvoir intervenir, il est indispensable de raisonner en terme de causalité. Nous avons vu que la phase comparative ne nous permet en fait, que de faire des hypothèses sur la causalité des liaisons mises en évidence. Pour s'assurer de la réalité de ces hypothèses, il est indispensable de passer par la démarche expérimentale.

\section{III. - MÉTHODE EXPÉRIMENTALE}

\section{1. - Expérimentations par modification des caractéristiques du milieu}

Sur le terrain, nous pouvons facilement modifier certaines caractéristiques chimiques du sol. Par contre, il est à peu près impossible de modifier la plupart des caractéristiques physiques, ce qui rend la vérification des hypothẻses à peu près impossible. II n'est donc pas étonnant que, sur peuplements adultes, les principales expériences de modification du milieu aient trait à la fertilisation. 


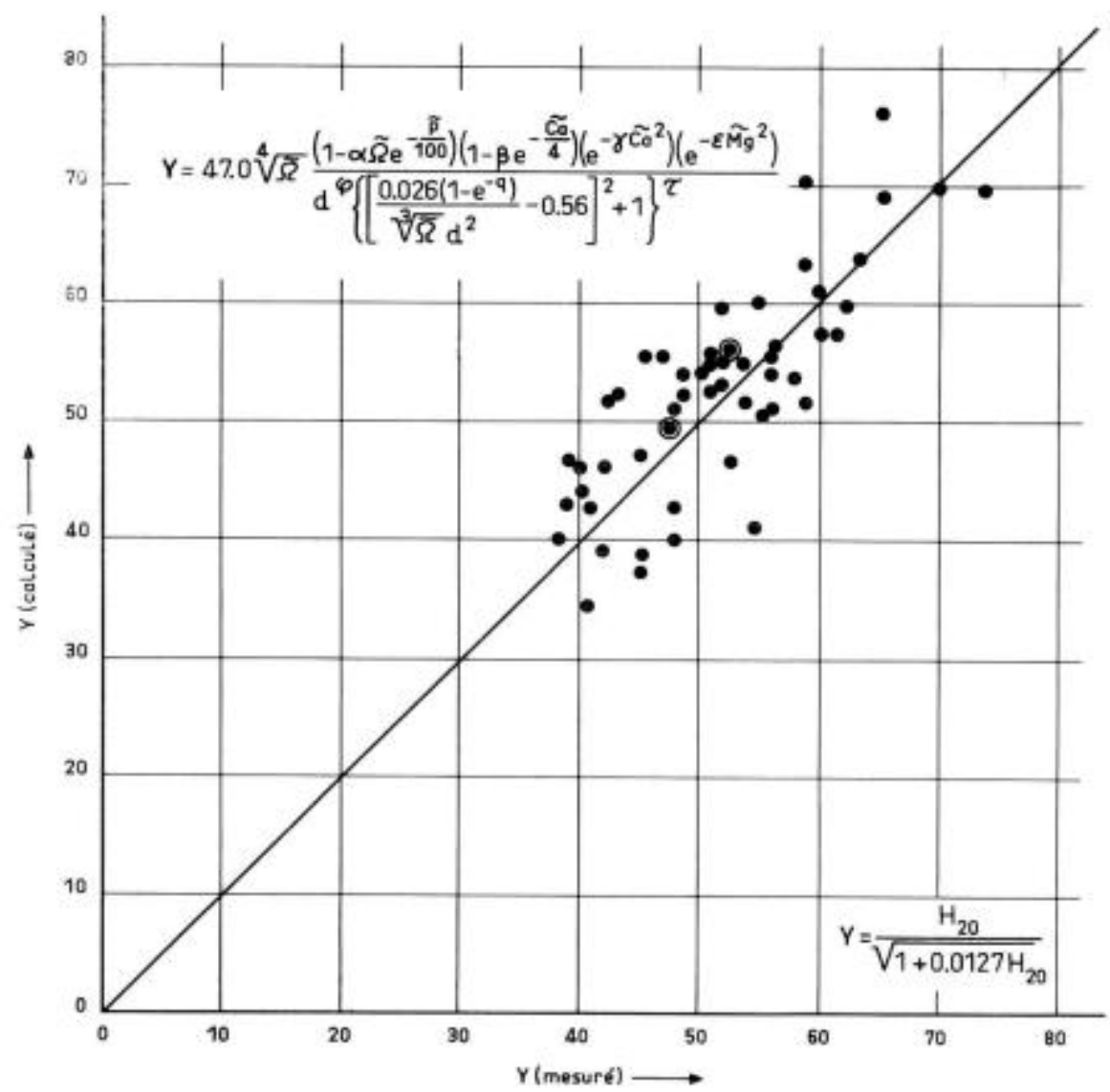

FIG. 5. - Comparaison entre les valeurs de y mesurées et les valeurs de y calculées par léquation de prédiction. Les valeurs de y peuvent étre transformées en hauteur dominante à 20 ans. Q Volume da sol utile. P teneur en phosphore total pon tévé en fonction de la profondeur $(\mathrm{ppm})$. Ca tenesur en calcium échangeable pondéré en fonction de la profondeser (en milliéquivalents pour $100 \mathrm{~g}$ ). $\mathrm{Mg}$ teneur en magnésium échangeable pondéré en fonction de la profondeur (en milliéguivalents pour $100 \mathrm{~g}$ ), d moyenne des particules du sol. $\mathrm{q}$ index climatique calculè à partir de la plaviosité et de plasieurs autres paramètres climatiques, e base des logarithmes naturels, $x, \xi, \gamma, z, \phi, \tau$ constantes calculées pour diverses stations et dépendant des conditions climatiques, dapress Carnowski M.S., Humphrey F. R., Gentle S. W., 1967

FiG. 5. - Comparaison between empirically obrained values of $\mathrm{Y}$ and computed ones by equation $(10)$ (the values of $\mathrm{Y}$ can be recomputed into site indices $\left(\mathrm{H}_{20}\right)$ by formala ( 9 ) Q2 effective upright-soil ratio, $\mathrm{P}$ - effective $\mathrm{P}$ in ppm, $\mathrm{Ca}$ - effective $\mathrm{Ca}$ in me/100 g, $\mathrm{Mg}$ - effective $\mathrm{Mg}$ in me/100 g, d - mean size of soil particles, q - humidity index, $\mathrm{e}$ - base of natural logarithms, $x, \beta, \gamma, z, q, 7$, - constant values for particular localities, depending on climate conditions) (Zarnowski M.S., Humphrey F.R., Gentle $S$. W. 1967).

Voici deux exemples de telles expériences. En bavière, une enquête préalable sur Épicéa avait montré l'insuffisance de la nutrition azotée en général et une liaison entre production et nutrition azotée. Dans trois peuplements présentant deux niveaux différents de nutrition azotée, KREUTZER a utilisẻ divers types de fertilisation.

Sur la figure 6, KREUTZER a porté, en abscisse, la teneur en azote des aiguilles d'Épicéa et, en ordonnée, l'accroissement courant $\mathrm{en}^{3} / \mathrm{ha} / \mathrm{an}$. Le peuplement ayant la plus mauvaise 
croissance a une teneur en azote de 1,2 p. 100 environ. Un apport de phosphore et de calcium n'a aucun effet; par contre, un apport d'azote permet de faire passer la teneur en azote des aiguilles à 1,45 p. 100 et d'obtenir un gain en accroissement courant par ha et par an de l'ordre de $4 \mathrm{~m}^{3}$. Sur les deux autres peuplements de plus haute fertilité, l'action d'un apport d'azote a les mêmes effets. Par fertilisation azotée, la teneur en azote des aiguilles passe de 1,4 à 1,6 p. 100 ce qui entraîne en gain en accroissement courant de l'ordre de $3 \mathrm{~m}^{3}$ environ par ha et par an.

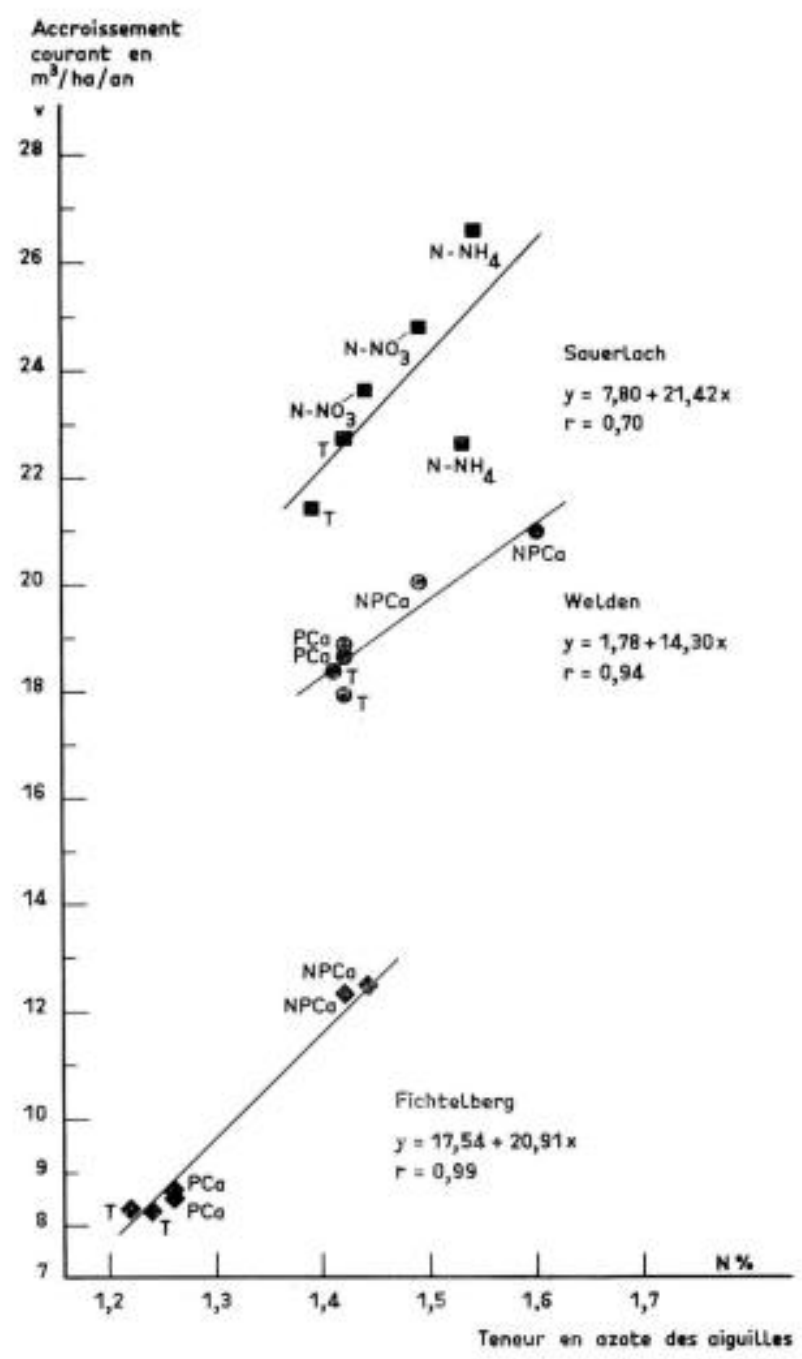

FIG. 6. - Liaison entre teneur en azote des aiguilles de Tèpicéa ef accroissement courant on $m^{3} / h a / a n d$ 'apres $K$ reutzer (1967)

Fici. 6, - Correlation between nitrogen content of spruce needles and anmual volume increments in cubic mefers per hectar (Krestzer 1967) 


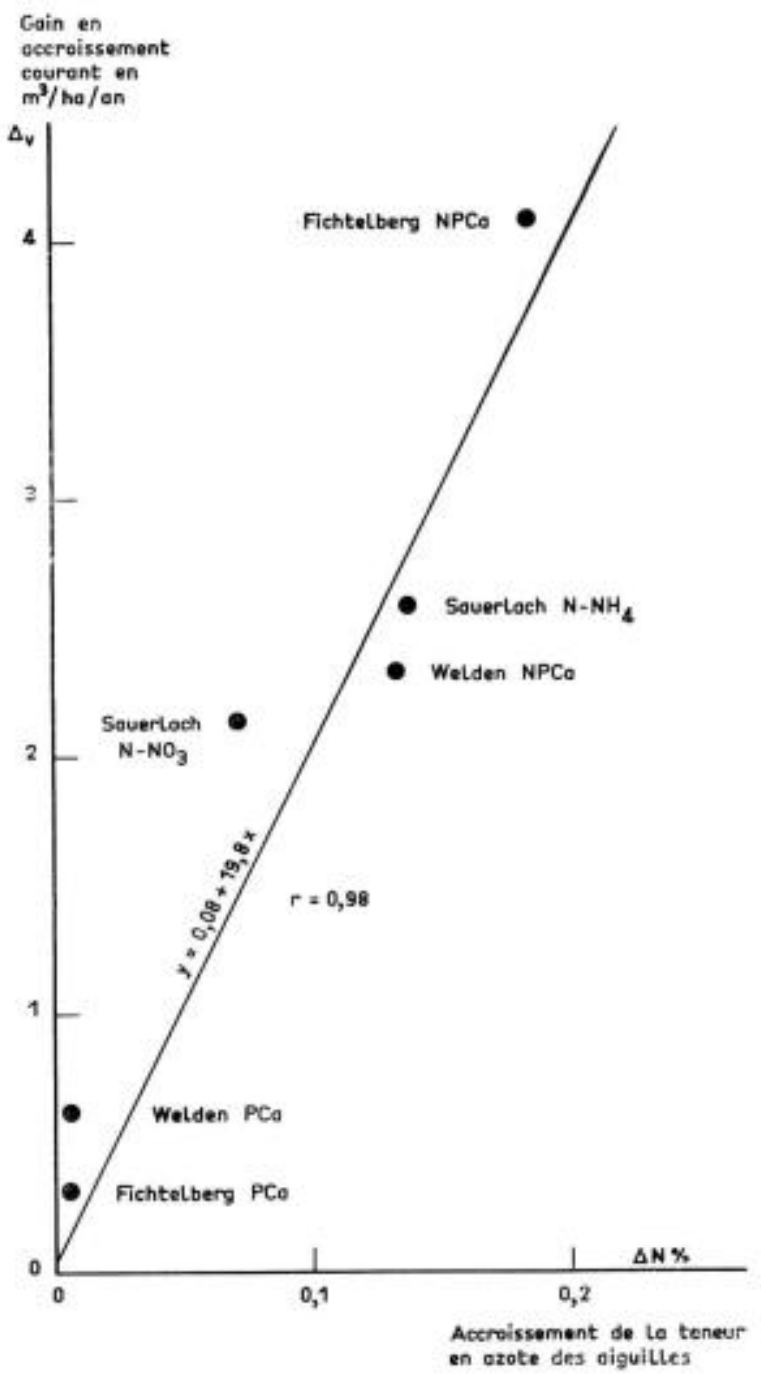

FiG. 7. - Liaison entre accroissement de la teneur en azote des aiguilles et gain en accroissement courant en $m^{3} /$ hajan d'aprèx Kreatzer (1967)

Fic. 7. - Correlation between increase of nitrogen content of spruce necilles and increase annual volume increment (Kreutzer 1967)

De ces divers essais, KREUTZER a pu tirer une loi plus générale résumée par le graphique 7. Ce graphique donne les gains en accroissement courant en fonction de l'augmentation de la teneur en azote des aiguilles. En gros, un accroissement de la teneur en azote des aiguilles de 0,1 p. 100 (moyenne des analyses de 1961, 1963 et 1965) correspond à un gain en accroissement courant de l'ordre de $2 \mathrm{~m}^{3}$ par ha et par an sur peuplements adultes en place. 
Un autre exemple intéressant peut être pris dans le travail de Leroy, repris maintenant par Garbaye, sur le Chêne dans l'Ouest de la France (Leroy, 1968). Au cours d'une enquête préalable, LEROY, avait constaté une déficience en $\mathrm{N}$ et $\mathrm{Ca}$ dans certaines stations et une bonne liaison entre la nutrition en ces deux éléments et la production. Il a tenté de vérifier la réalité de cette liaison en installant un dispositif sur gaulis à Bercé. Après trois ans, voici les résultats (communication personnelle).

\begin{tabular}{|c|c|c|c|}
\hline Traitements & o & $\mathrm{NCa}$ & $\mathrm{NPKCa} \mathrm{Mg}$ \\
\hline $\begin{array}{l}\text { Accroissement moyen sur le rayon pendant } \\
\text { la période de } 3 \text { ans (en } \mathrm{mm}) \ldots \ldots \ldots \ldots \ldots\end{array}$ & 4,02 & 5,64 & 5,49 \\
\hline
\end{tabular}

Ces résultats confirment parfaitement l'hypothèse de départ issue de l'enquête préalable. On pourrait ainsi multiplier de tels exemples.

Cette façon de travailler permet de déterminer les véritables liaisons de cause à effet et donc d'intervenir par modification de certaines caractéristiques du milieu. Mais si I'on peut déterminer quel facteur agit sur la production, on ne sait pas comment il agit. Or, it peut être extrêmement intéressant de connaitre ces mécanismes d'action, ne serait-ce que pour se donner de nouvelles possibilités d'intervention.

Nous en arrivons ainsi aux expérimentations à caractẻre physiologique ou écophysiologique.

\section{2. - Expérimentations à caractères physiologiques ou écophysiologiques}

Certaines techniques permettent de travailler sur l'arbre adulte, ce qui est extrêmement intéressant. L'utilisation d'éléments marqués rend de grands services et nous en avons de nombreux exemples. L'utilisation d'engrais marqués, pour ne prendre que ce type d'exemple, permet de savoir ce qu'un élément devient dans le sol, de savoir à quelle vitesse il disparait, soit par lessivage, soit par rétrogradation, et ả quelle vitesse il est absorbé par l'arbre et dans quelle proportion. On conçoit facilement que la connaissance de ces mécanismes améliore finalement nos possibilités d'intervention.

Mais la plupart du temps, il est difficile de poursuivre des investigations sur arbre adulte pour des raisons faciles à comprendre.

Nous sommes donc très souvent amenés, par la force des choses, à travailler sur de jeunes plants ou de jeunes semis.

Immédiatement se pose le problème de la liaison entre le comportement du jeune plant et de l'arbre adulte. Il est difficile de savoir si, d'une manière générale, ce comportement juvénile est différent de l'adulte, mais nous avons plusieurs exemples qui doivent nous inciter à la prudence. Nous en citerons deux concernant l'Épicéa et le Pin sylvestre. L'Épicéa est très calcifuge à l'état juvénile, mais, avec le temps, sa tolérance au calcaire s'améliore nettement. Le Pin sylvestre a un comportement exactement inverse. A l'état juvénile, il est trés tolérant et se comporte remarquablement, même sur les sols les plus calcaires. Par contre, au bout de quelques dizaines d'années, il manifeste des signes de défaillance qui se marquent par une chlorose généralisée. 
Le mécanisme d'action du calcaire du sol sur les résineux, dont beaucoup présentent une chute de production dès qu'il est présent dans tout le profil, est intéressant à considérer et peut illustrer l'intérêt que nous avons à connaitre certains mécanismes écophysiologiques.

Le carbonate de calcium agit sur les résineux de manière indirecte en modifiant de nombreuses propriétés du sol, mais aussi directement. Cette action directe se fait au niveau de la rhizosphère. Les racines exsudent dans le sol divers acides organiques, ce qui entraîne un double phénomène au niveau des racines : d'une part, une augmentation considérable de la concentration en ion calcium provenant de la dissolution du calcaire par les acides organiques, ce qui est néfaste, et d'autre part, un abaissement du pH, ce qui est favorable.

Il semble que ce soit ce deuxiẻme effet qui l'emporte, d'oủ l'idée d'augmenter la production d'acides organiques par les racines pour améliorer la résistance au calcaire.

Un Britannique, JoNes (1971), vient tout récemment de démontrer que l'on peut améliorer cette production d'acides organiques par les racines en appliquant un produit, la choline, à des concentrations très faibles. II a ainsi obtenu des améliorations de rendement de 100 à 150 p. 100 de plants calcifuges en milieu calcaire. II n'est pas impossible que l'on puisse obtenir des résultats identiques sur Épicéa, ce qui serait très intéressant pendant les premières annćes oủ il est particulièrement sensible au calcaire.

\section{IV. - CONCLUSION}

Cette approche écophysiologique nous permettra probablement d'effectuer, dans les années à venir, des progrès insoupçonnables dans la connaissance des mécanismes d'action du sol sur le végétal. Mais les résultats pratiques que l'on peut en attendre sont aléatoires et certainement pas envisageables à court terme sauf exception. Ce type de recherches prendra le relais des autres voies d'approche lorsque nous aurons épuisé toutes leurs possibilités.

Les résultats que l'on peut attendre de la voie expérimentale par modification des conditions de milieu sont d'une toute autre portée à court terme. Il suffit de songer aux possibilités d'intervention que cela a entraîné dans le domaine de la fertilisation.

Quant à la méthode comparative, dont le but est de rationnaliser le choix de l'essence, elle est probablement arrivée ả un stade d'élaboration proche de la limite de ses possibilités. Des améliorations sont encore possibles (amélioration des mesures des caractéristiques de nutrition ou de sol, amélioration des techniques d'interprétation mathématiques), mais elles n'en augmenteront plus l'efficacité de manière importante.

Cependant, malgré ses imperfections et ses limites, si elle était appliquée de manière systématique, la méthode comparative pourrait rendre, à son stade actuel, des services considérables.

\section{SUMMARY}

\section{SOIL, NUTRTIION AND WOOD PRODUCTION}

For a long time, foresters have been trying to know how soil could influence the growth of forests.

The first attempts have been done indirectly, by using vegetation. Since, this method has been considerably improved. At the same time, the research of direct relations between production and 
soil characteristics, without using vegetation, has been developped from 1920 and mostly from 1940.

Two methods can be used :

The first one gives soil-production relationships, without looking for causality; it is the comparative method, or survey.

The second one, on the contrary, explains the influence of soil on tree by experimentation (often eco-physiologic), with the control of one or several factors.

In the comparative method, we choose a number of forest-site representing all the sorts of soils in a region with the same climate. On each site, we make two sorts of measurements :

- production measures.

- measures concerning soil. These ones can be indirect (foliar analysis). The problem is to establish relations between these two sorts of measurements; three methods can be used :

- simple methods, with the use of variance analysis with one controlled factor.

- methods with the use of the correlation coefficients (multivariate analysis).

- methods with the use of non-linear models.

The second and third methods need a powerful calculating device (computer). This comparative method has now reach a great efficiency, near of the limit of its possibilities.

Meanwhile, in spite of its numerous imperfections, it could now help to predict the wood production of news stands or to choose species for reforestation, upon condition of using it systematically.

In the experimental method, one can modify the soil, or try to understand the way soil characteristics influence the tree (eco-physiology).

It makes possible to discover causal relations between soil and forest, and therefore to modify some soil characteristics to improve production. The development of forest fertilisation is a direct consequence of this sort of research.

The eco-physiologic way of research will probably improve our knowledge of soil tree relationships. But the technical results which may occur are uncertain and will not be usefull before a long time.

This sort of research will certainly replace the other methods when their possibilities are exhausted.

\section{RÉFÉRENCES BIBLIOGRAPHIQUES}

Aussenac G., 1970. - Action du couvert forestier sur la distribution au sol des précipitations. Ann. Sei, forest., 27 (4) 383-399.

Aussenac G., BeCKER M., 1968. - Écologie d'un massif sur sols hydromorphes. La forêt de Charmes (Vosges). Contribution à la mise au point d'une méthode dynamique du milieu forestier. Ann. $S c i$. fore'st, (4).

BAzUki M., 1971. - Outline of systems analytic approach to forest growth and site evaluation. IUFRO XV Congrès, Gainsville U.S.A.

BECKER M., 1969, - Le hêtre et ses problèmes en forềt de Villers-Cotterêts. Contribution à la mise au point d'une méthode d’étude écologique du milieu forestier. Publication du Laboratoiré de Botanique forestière, C,N.R.F. Nancy. Ann. Sci. forest. (2).

Blum W. E., 1971, - Correlations between nutritive elements in roots and needles of Norway spruce (Picea abies) in relation to the nutritive status of soil. Contribution to site factors and plant nutrition, IUFRO XV Congrès, Gainsville U.S.A.

Bonneau M. 1967, - Minéralisation de l'azote de deux sols des Hautes Vosges allure du phènomène. Sci, sol (1),

Bonneau M., Gelpe, Le TACON F., 1968. - Influence des conditions de nutrition minérale dans le dépérissement du pin maritime dans les Landes de Gascogne. Ann. Sci. forest., 25 (4).

CAsAnder A. K., 1933. - Untersuchungen über die Entwickungen der Kulturfichtenbestände in Süd Finnland. Commt. Inst. Forest. Fenn, 19-3.

Cajander A. K., 1949, - Forest types and their signifigance. Acta. For. Fenn. 56.

Carmean W. H., 1954, - Site quality for Douglas Fir in Southwestern Washington and its relationship to precipitation elevation, and physical soil properties. Proc. Soil Sci. Amer, 18, 330-334.

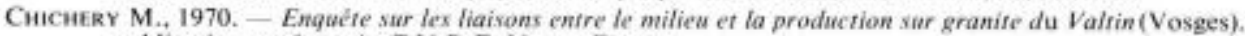
publication ronétypee C.N.R.F. Nancy France.

Conte T. S., 1938, Classification of forest sites with special reference to ground vegetation. Journ. For. 36, 1062-1066. 
Coule T. S., 1948. - Relation of soil characteristics to site index of loblolly and shortleaf pines in the lower Piedmont region of North Carolina Duke, Univ. School of Forestry, Bull., 13, 78.

Conle T. S., Schumacker F. X., 1953, - Relation of soil properties to site index of loblolly and shortleaf pines in the Piedmont region of the Carolinas, Georgia and Alabama. Journ. For., 51, 739-744.

Czarnowskı M. S., 1964. - Productive capacity of locality as a function of soil and climate with particular reference to forest land. Baton Rouge, La. (U.S.A.), 189.

Czarnowski M. S., Humphleys F. R., and Gentle S. W., 1971. - Quantitative Expression of productivity in Moaterey Pine plantation in Australie and New-Zeeland in terms of some soil and climate characteristics. IUFRO XV Congress Gainesville, U.S.A.

Czarnowski M. S., Humphrey F. R., and Gintle S. W.. 1967. - Site-Index as a function of soil and climatic characteristics fa preliminary note based on man-made stands of Pinus Radiata D.DON. in New South Wales, Australia). Ekologia Polska, tom. XV, n" 23, 495-504.

Dagnelif D., 1956-1957, - Recherches sur la productivité des hêtraies d'Ardennes en relation avec les types phytosociologiques et les facteurs écologiques, Bull. Inst. Agron. Sta. Rech. Gembloux, 24. $249-284,25,44-94$.

Donahue R. L., 1940. - Forest site quality studies in the Adirondacks : I. Tree growth as related to soil and morphology. Cornell. Univ. Agric. Expt, Sta. Mem., 229, 44.

Duchaufour Ph., Alt., 1958. - Un exemple d'utilisation pratique de la cartographie des stations : la forêt du Ban d'Etival (Vosses). R.F.F., 10, 597-6.30.

Decoukt N., Godkon M., Romane F, Tomassove R., 1969, - Comparaison de diverses méthodes d'interprétation statistique de liaison entre le milieu et la production du Pin sylvestre en Sologne. Ann. Sci. Forest., 26, (4), 413-443.

Decourt, Le Tacon, 1970, - L'épicéa commun sur les plateaux calcaires de l'Est de la France. Essai de prévision de la production à l'aide de déterminations pédologiques simples. Ann. Sci. forest.. 37 (3), 255-286.

Fliche P. et Grandeau L. 1873. - De I'Influence de la composition chimique du sol sur la végétation du pin maritime (Pimus pinaster) Annales de chimie et de physiąue, 4 e série, $\mathrm{t}$. XXIX, 1-32.

Fourt D. F., Donald D. G. M., Jeffers J. N. R., and Binns W. O., 1971. - Corsisan Pine (Pinus nigra var. maritima (Ait) Mel.vit..E) in Southem Britain. A study of Growth and Site factors. Foresfry. XLIV, 2.

Gasser R. N., 1950, - Relation between soil characteristics and site index of Loblolly. Pine in the coastal plain region of Virginia and the Carolina, Journ. For., 48, 271-275.

Garbaye J., Leroy Ph., Le Tacon F., Levy G., 1970, - Réflexions sur une méthode d'etudes des relations entre facteurs écologiques et caractéristiques des peuplements. Ann. Sci. forest., 27, (3) 303-321.

Garbaye J., Leroy Ph. Oswald H. - Premiers résultats de cinq années de fertilisation sur jeunes peuplements de chène en forêt de Bercé : 1974, Revue forestière française (sous presse).

Gessez S. P., 1949. - Correlation between certain soil characteristics and site for Douglas Fir in northwestern Washington. Proc. Soil. Sci. Soc. Amer., 14, 333-337.

Gesset S. P., Lloyo W. J., 1950. - Effect of some physical soil properties on Douglas Fir site quality. Journ. For., 48, 405-410.

Gessel S. P., 1967, - Concepts of forest productivity, XIV IUFRO Congress, Münich II, 36.

Geborkiantz S. R., Scholz H. F., 1944. - Determining site quality in understocked oak forests. Journ. For., 42, 808-811.

HARTMANN F. K., 1926. - Die Abhångigkeit der Hohenbonitãt und der Bodenflora der Kiefer yom Feinerdegehalt und Untergrund gewisser diluvialer Sansbə̌den. Zeitschr. Forst u Jagdw, 58. 226-255.

JONES WYN R. G., 1971. - A possible new method for improving plant growth in saline and calcareous environments. Symposium on the use of isotopes and radiation in research on soil-plant Vienna 13-17 décember 1971 .

ILvessalo Y., 1923, - Ein Beitrag zur Frage der Korrelation zwischen den Eigenschafien des Bodens und dem Zuwachs des Waldbestendes. Acta Forest Fenn., 25, 31.

JONES JOHN R., 1969. - Review and comparison of site evaluation methods. UDSA Forest Service Research Paper R M-SI, 27 p. Rocky Mountain Forest and Range experiment Station, Fort Collins, Colorado 80521.

Korstian C. F., 1917. - The indicator significance of native vegetation in the determination fo forest sites. Plant World, 20, 267-287.

Korstiav C. F, 1919, - Native vegetation as a criterion of site, Plant World, 22, 253-261.

Kreutzer K., 1967. - Ernährungszustand und Volumenzuwacks von Fichtenbestanden neuer Düngungsversuche in Bayern. Forstw. Cbl., 86, 176.

LEMéE. G., 1967. - Investigations sur la minéralisation de Pazote et son évolution annuelle dans les humus foresticrs in situ Oecol. Plant. Vol. 2, 285-324.

Leroy Ph., 1967. - Variations de productivité du Chêne sur les pentes á Bercé. Nécessité d’une cartographie des sols. R.F.F., $\pi^{\prime \prime} 40$.

Leroy Ph., 1968. - Etudes de nutrition et production sur le chène, perspectives de fertilisation. R.F.F., n०5. 
LE TACON F., 1968. - Un essai d’application de la cartographie des sols. Nécessité de la connaissance des facteurs de production. R.F.F., $\mathrm{n}^{\circ} 4$.

LE TAcon F., Oswald H., 1969, - Les sols en Haute-Ardéche. Caractérisation et production forestière. Bulletin Association française pour l'Étude du Sol.

Le Tacon F., Oswald H., Tomassone R., 1970. - La nutrition minérale de l'Épicéa en Haute-Ardèche. Liaison avec la production. Ann. Sci. forest., (4).

Le Tacon F., Muller C., 1970. - Influence des conditions de nutrition minèrale sur la croissance de l'épicéa commun (Picea excelsa) sur les plateaux calcaires de l'Est de la France. Amn. Sci. forest., (4).

LE TAcon F., MiLuer C., 1971, - L'influence des caractéristiques du sol sur le comportement des essences forestières. Exemples et critiques de méthodes d'interprétation mathèmatique. IUFRO Congress $X V$, Gainesville U.S.A.

Levy G., 1967. - Influence de certaines propriétés du sol sur la densité d'enracinement de l'épicèa commun et du pin sylvestre. Thèse Docteur-Ingénieur, Faculté des Sciences, Nancy.

LEvy G., 1969. - Premiers résultats d'étude comparèe de la nappe temporaire des pseudogleys sous résineux et sous feuillus, Ann. Sci. forest ., 36 (1), 65-79.

Lowry G. L.. 1964. - Forest Soil Sie Studies I. Objectives, Sampling. preliminary results on black spruce P.P.R.I.C. Technical reports, no 368 .

LUNT H. A., 1939, - Soil characteristics topography and lesser vegetation in relation to site quality of second-growth oak stands in Connecticut. Journ, aer, Res., 59, 407-428.

MANer D. L., 1971. - Evaluation of some problems in the multifactor approach to site, IUFRO XV Congress, Gainesville U.S.A.

Mitschfrlich E., 1909. - Das Gesetz des Minimus, das Gesetz des Abnahmenden Bodenertrages. Landw. Jahrb. 38, 537-552.

MıтschғRLıch E., 1916. - Pllanzenphysiologie Vorarbeiten zur chemischen Düngelmittelanalyse. Landw. Jahrb. 49, 335-342,

Miтschғrlich E., 1916, - Zum Gesetz des Pflanzenwachstums. Landw. Jahrb. 49, 167-183.

Mitscherlich E., Duhring F., 1926. - Das Liebigsche Gesetz von Minimum und das Wirkungsgesetz der Wachstumfaktoren. Ahh. Königsberg Gelehrte Ges. Naturwiss 3, 1-30.

Mitscherlich E., Duhring F., 1928. - Uber die Konstante im Wirkungsgesetz des Wachstums factoren. Abh. Künigsberg Gelehrle Ges. Naturwiss 5, 17-40.

Myers C. A., Deasen J. L. van, 1960. - Site index of Ponderosa Pine in the Balck Hills from Soils and topography, J. For, 58, (7).

Nys C., 1969, - Croissance du hẽtre sur les sols de la forết de Haye. Diplôme d'éfude approfondie. Faculté des Sciences de Nancy.

PAGE G., 1970, Quantitative Site Assessment : Some Pratical Applications in British Forestry. Forestry, 43, 1, 45-56.

PAGE G., 1971. - Evaluation of interrelationships between site conditions and the growth of fir and spruce in Newfounland. IUFRO XV Congress, Gainesville U.S.A.

Oswald H., 1969, - Les potentialités forestiéres en Haute-Ardèche, l'épicéa commun. (Picea Abies L.) Ann. Sci. forest., 26 (2), 183-224.

PARde J., 1961. - Dendrométrie. 360 p. Imprimerie Louis-Jean, Gap.

Reнruess K. E., 1971. - Einige Entwicklungslinien und Aufgaben der forstokölogischen Forschung in Süddeutschland. Forstw. Cbl. 90, p. 349-363.

RENNIE P. J., 1963, - Methods of assossing Forest site Capacity. Commonwealh forestry Review, vol. 42. (4), n० 114, décembre 1963.

RıGG G. B., 1929. - Using the vegetation cover as an aid in studying logged-off as forest sites. Journ. For., 27, 539-545.

Rowe J. P., 1956. - Classification of site for forest management Int. Union of forest. Res. Org, 12 th Congr. Oxford.

Row C., 1960, - Soil Site relations of old fuld, Slash Pinc plantations in Carolina sans hills, F. For., 58, (9),

SEAL H., 1964. - Multivariate analysis for biologists. Methuren - Londres.

Splls Bulri R. H., Sмrru D. A., 1957. - Forest site of the pacific Northwest Canada, Ministère des Terres et des Forêts. Service Forestier.

SteCKeler J. H., 1948. - The growth of Quaking Aspen as affected by soil properties and fire. Journ. For, 46, 727-737.

TAMm O., Wadma E., 1945. - Sur les facteurs stationnels et leur corrélation avec les conditions forestières dans le district de Hamra (Texte suédois) Svenska Skogs., Tidskr., Bihang, 2, 79 p.

TАмм C. O., 1971. - Nature's laws or pure empiricison? Site studies as a guide to better use of forest land. IUFRO XV Congress, Gainesville, U.S.A.

TARrant R. F., 1949. - Douglas-Fir site quality and soil fertility, Journ. For., 47, 716-720.

Tomassone R., 1965. - L'analyse des composantes principales. Publication ronéotypée de la Station de Biométrie, C.N, R.F. Nancy. 
Tomassone R., 1965. - Analyse des régressions progressives. Publication ronéotypée de la Station de Biométrie. C.N.R.F. Nancy.

Tomassone R., 1967. - Regression multiple progressive. Station de Biométrie. C.N.R.F. Nancy. Programme A. G. 65007 .

Tomassone R., 1967, - Une méthode d'investigation : la régression orthogonale. Ann. Sci. Forest., 24. (3), 233-258.

VALLEE G., 1967. - Utilisation des relations entre la végétation du sous-bois et la productivitè des stations pour la classification des foréts. Thèse Doct. Ing. 2e sujet. Fasc. Sciences de Nancy.

VAN Goor C. P., LABastide J. G. A., 1970. - Growth-site relationships in plantations of Pinus Elliotii and Araucaria aygustifolia in Brazil. Plant and Soil, 32, 349-366.

Vıro P. J., 1947, - La composition granulométrique et la fertilité des sols forestiers en considèrant principalement la pierrosité (Texte finlandais). Commun. For. Fenn., 35, 115.

Werner H., 1964. - Grundzüge einer regionalen Bodenkunde des Sãwestdeutschland Alpenvorlandes. Schriftent. Landesforstverw. Baden Würt. 17.

Werner H., 1962. - Untersuchungen uber das Wachstum des Hauptholzarten auf den wichtigsten Stanortseinheiten der Mittleren Alb. Mitt. Ver. Forstl. Standortskds, y. Forestpflanzenzüchfung, 12.

Youngererg C. T., Scholz H. F., 1949. - Relation of soil fertility and rate of growth of mixed oak stands in the Driftless area Southwestern Wisconsin. Proc. Soil. Sci. Soc. Amer., 14, 331-332.

\section{DISCUSSIONS}

M. Decourt. - La hauteur dominante n'exprime pas la "productivité potentielle » de la station puisqu'elle intègre également les potentialités du matériel végétal utilisé.

M. LE TACON. - Il ne faut pas en effet l'oublier, mais cela est toujours présent dans I'esprit de ceux qui entreprennent des études sol-production. On peut d'ailleurs estimer, dans le cas des peuplements artificiels, que la part due au potentiel génétique dans l'explication de la variation de hauteur dominante, se retrouve dans la variation résiduelle, c'est-à-dire dans la variation non expliquée par les variables du sol prises en compte.

M. Bonneau. - La méthode comparative, si elle donne parfois des résultats limités, est néanmoins toujours utile sinon indispensable. En effet, même si l'explication de la production que l'on peut ainsi obtenir est faible, elle permet de collecter des données et des connaissances très utiles. Il peut par exemple arriver qu'un facteur peu variable limite la production. il n'apparaitra donc pas comme facteur explicatif de la variation de production. Néanmoins, I'enquête permettra de se rendre compte que ce facteur se trouve à un niveau déficient et limitant et permettra donc d'orienter un programme d'expérimentation ultérieur.

M. Duchaufour. - En ce qui concerne l'étude des corrélations entre les diverses propriétés du sol et la production, il est évidemment indispensable d'effectuer une étude analytique, c'est-à-dire d'examiner séparément les liaisons existant entre les propriétés du sol et la croissance ou la nutrition. Cette étude sz traduit ensuite par l'établissement d'une ou plusicurs équations explicatives.

II ne faut pas oublier de compléter ce travail analytique par un travail de synthèse avec lequel pédologues et écologistes sont familiarisés : le sol est un milieu complexe qui intègre l'ensemble des propriétés étudiées; il convient de le définir par le type génétique, la profondeur, le matériel originel, etc. Le type de sol sera donc un élément essentiel de diagnostic pour le forestier compte tenu des analyses détaillées qui auront été faites préalablement.

M. LE TACON. - Dans tous les travaux consacrés aux études sol-production, nous 
essayons toujours de faire intervenir en premier lieu le sol en tant que facteur synthétique. La plupart du temps d'ailleurs, la phase d'interprétation débute par une méthode simple, l'analyse de la variance à un facteur contrôlé, oủ justement le facteur contrôlé est généralement un facteur synthétique tel que le type de roche-mère, le type de sol ou le type d'humus.

M. Eefkman. - Peut-on considérer qu'un pourcentage d'explication de 50 p. 100 obtenu à l'aide de six variables soit satisfaisant, même pour des prévisions?

M. Le TACON. - Plus le pourcentage d'explication est élevé, meilleures seront les prévisions de production. Il arrive que ce pourcentage d'explication atteigne 80 et 90 p. 100 pour certaines études étrangères, ce qui permet de prévoir la production sans risque d'erreurs ou presque. En France avec l'expérience que nous avons à l'heure actuelle, ce pourcentage d'explication ne dépasse guère 60 p. 100. C'est encore important quand on sait que de multiples autres facteurs que le sol interviennent dans l'explication des variations de production. Les fourchettes de prévision sont évidemment d'autant plus lâches que le pourcentage d'explication est plus faible. Mais même avec un pourcentage d'explication de l'ordre de 50 p. 100 , les possibilités de prévision sont encore acceptables, surtout si la variation de production est importante.

M. Oswald. - Le choix d'un échantillonnage systématique pour sélectionner les différentes stations introduit une variation supplémentaire et peut ainsi expliquer en partie la relative faiblesse de certains pourcentages d'explication.

Dans certaines études (Épicéa en Ardèche par exemple), on effectue un échantillonnage hiẻrarchisé c'est-à-dire en fonction d'un ou plusieurs facteurs apparaissant importants, ce qui permet semble-t-il d'obtenir des pourcentages d'explication plus élevés.

M. AYMonin, - La formule de Czarnowsky est intéressante. La signification des caractères a-t-elle été étudiée d'une façon corrélative par une analyse de leurs liens linéaires?

L'ordinateur fournit une réponse qui est évidemment fonction de ce qu'on lui donne à analyser. 11 serait done très précieux de savoir à quel niveau les divers caractères sont corrélés entre eux, dans l'hypothèse oủ ils n'auraient pas tous la mème valeur par rapport aux résultats obtenus dans l'application de la formule synthétique.

M. Millier. - Le modèle de Czarnowski est le contrepied des modèles linéaires classiques (régressions et analyses de variance). Il prend en compte deux remarques de base. c'est-à-dire qu'un élément peut être limitant et que, d'autre part, un élément peut être consommé de façon excédentaire sans influencer la production (ou bien être toxique).

En conséquence, les coefficients de corrélation simples n'ont pas tellement d'intérêt et ne peuvent servir qu’à une présélection des variables intéressantes.

M. Duchaufour. - Les diagnostics tirés de l'analyse foliaire me paraissent plus valables quand ils sont effectués sur des arbres jeunes, que s'il s'agit d'arbres adultes.

Dans la majorité des cas, les carences les plus graves interviennent dans les phases de jeunesse. Elles sont surmontées ensuite par le peuplement adulte qui a la possibilité d'améliorer sa nutrition en développant son système racinaire et en assurant la prospection de nouvelles couches de sol. On peut citer comme exemple la carence en azote des jeunes épicéas sur pelouse d'altitude ou l'influence néfaste du calcaire sur la nutrition des jeunes plants d'Épicéa, etc. 
M. LE TACON. - Il est vrai que certains phénomènes de carence sont liés au stade juvénile. Au moment de la plantation, le système racinaire est lésé et ne peut assurer immédiatement une nutrition convenable en éléments minéraux, comme d'ailleurs il ne peut assurer une alimentation en eau suffisante. Au bout d'une ou plusieurs années, suivant les essences, suivant les conditions de station ou les conditions climatiques, cette crise de transplantation est surmontée et la croissance redevient normale avec la disparition des troubles de nutrition. Il est exact également que certains phénomènes de carence en azote liés à la présence de calcaire, par exemple pour l'Épicéa, se manifestent surtout pendant les premières années puis s'estompent. Mais on peut citer des exemples inverses. D'autre part, actuellement, nous sommes en possession de nombreux résultats d'essais de fertilisation effectués un peu partout dans le monde, sur peuplements adultes. Ces essais montrent que les arbres adultes sont parfaitement capables d'absorber les éléments minéraux qui leur font défaut lorsqu'on les leur apporte, et sont capables de réagir à cette absorption par une amélioration de leur production. Les graphiques donnés par KREUTZER sont très significatifs à cet égard et ne constituent pas des résultats isolés.

M. DeCOURT. - A quelle mesure est rapporté le résultat des analyses foliaires (100 grammes biomasse des feuilles, etc.)? La signification doit être différente suivant les cas.

M. LE TACON. - On exprime généralement les résultats des analyses foliaires en p. 100 de matière sèche. Si l'on pouvait rapporter ces résultats à la biomasse des peuplements, cela permettrait, entre autres, d'éliminer les phénomènes « parasites " de concentration ou de dilution des éléments dans la masse foliaire.

M. Bonneau. - Il est possible d'éliminer, en partie, l'inconvénient d'exprimer les éléments intervenant dans la nutrition en p. 100 du poids sec, en faisant intervenir le poids moyen des feuilles ou des aiguilles, ce qui donne un indice de la quantité totale d'éléments consommés par l'arbre.

M. Schvester. - A propos des mérites ou des défauts comparés de l'analyse foliaire et de l'analyse racinaire, ne peut-on pas penser que l'analyse foliaire est plus intégratrice et reflète mieux (en dépit de ses défauts), l'ensemble des conditions de nutrition que l'analyse racinaire effectuée sur des prélèvements forcément localisés dans la rhizosphère?

M. Riedacker. - Le diagnostic foliaire est le reflet à la fois des réserves minérales du sol et du stade physiologique ou de certains mécanismes physiologiques de la plante. Par exemple des Eucalyptus ayant des systèmes racinaires de même âge, mais traités les uns en taillis et les autres en futaie sur une même station, ont des teneurs minérales fort différentes. C'est ainsi que la teneur en phosphore des feuilles du taillis exprimée en p. 100 du poids sec est de 50 p. 100 supérieure à celle des arbres traités en futaie.

On a pu montrer que ces écarts étaient dûs semble-t-il à des différences de vitesse de transport dans la tige.

II se pourrait, par conséquent, que l'analyse racinaire fournisse un meilleur indice relatif aux réserves minérales du sol que l'analyse foliaire puisqu'elles permettraient de s’affranchir de certains phénomẻnes physiologiques perturbateurs.

M. JANIN. - A propos de la fertilisation du Chêne, il serait important de vérifier si 
l'accroissement en diamètre lié à l'augmentation de la largeur de l'aubier est suivi d'une augmentation proportionnelle du bois de cour.

C'est un autre aspect de la physiologie de l'arbre qui se rapporte à la qualité du bois produit. II s'agit en effet de mieux connaitre la vitesse de duraminisation du chêne.

M. BONNEAU. - Le problème de l'influence de la fertilisation sur la qualité du bois de chêne de tranchage est particulièrement importante. On peut faire remarquer que dans la plupart des chênaies de haute qualité du Centre et de l'Ouest de la France, les gestionnaires n'arrivent pas, dans le temps fixé, à obtenir le diamètre d'exploitabilité voulu $(80 \mathrm{~cm}$ à 180 ans). La fertilisation peut donc être un moyen d'y parvenir. D'autre part, les essais actuels portent sur des gaulis et intéressent done un bois qui ne sera pas tranché. Enfin ces essais sont trop récents pour pouvoir se faire une opinion sur l'influence de la fertilisation sur l'augmentation respective de l'aubier et du bois de cœur.

M. Perrier. - Les augmentations de production annuelle constatées chez des peuplements d'Épicéa à la suite des expériences de fertilisation qui ont été présentéss (apports d'azote et de phosphore) semblent tout à fait spectaculaires. II serait intéressant de savoir :

- si ces augmentations ont été de courte durée ou de longue durée, et à la suite de quels apports effectués à quel rythme,

- en bref, de savoir si de telles augmentations peuvent être obtenues sur la majeure partie de la vie d'un peuplement, à la suite d'interventions suffisamment légères et suffisamment espacées dans le temps pour qu'elles demeurent acceptables sur le plan de la rentabilité.

M. Bonneau, - L'azote est de très loin l'élément le plus efficace en fertilisation des peuplements adultes. La réponse à l'azote est proportionnelle à la dose appliquée jusqu’à 200 ou $300 \mathrm{~kg}$ par hectare. Suivant la fertilité initiale de la station, les gains en volume sont compris généralement entre 10 et 60 p. 100 et parfois plus. La réponse à l'azote dure 5 à 10 ans et peut être obtenue jusqu à des âges avancés.

Des apports d'autres éléments tels que le phosphore ou le calcium ont, d'une manière générale, une efficacité bien moindre mais ont des effets plus durables.

M. Mérro. - Dans les pays scandinaves, la rentabilité économique et financière des interventions de fertilisation n'est, le plus souvent, assurée que lorsque des interventions ont lieu en fin de rotation.

Pour ce qui concerne les résultats obtenus à cet égard en R.D.A., il est difficile d'en comparer la valeur économique et financière avec celle des résultats techniques homologues obtenus dans un autre style d'économie.

M. LANIER. - Quels sont les types d'acides organiques « exsudés $n$ as niveau de la rhizosphère, et ceux-ci proviennent-ils de la racine elle-même ou des micro-organismes de la rhizosphère? L'addition de choline peut également agir par l'intermédiaire de la microflore du sol.

D'où la nécessité d'étudier l'influence des racines seules en milieu stérile.

M. LE TACON, - Les acides organiques exsudés au niveau de la racine sont les mêmes que ceux présents dans les tissus racinaires. Divers auteurs ont ainsi identifiés les acides 
oxaliques, critiques, maliques, glycoliques, succiniques, fumariques, pyruviques, etc... ainsi que de nombreux acides aminés comme la leucine, la valine, l'acide glutamique, l'ornithine, l'arginine, la tyrosine, etc. Il est certain que de tels acides peuvent provenir également des micro-organismes du sol et, de plus, les micro-organismes du sol peuvent influer sur la production d'acides organiques ou d'acides aminés des racines de plantes supérieures.

M. Delatour. - Il semble que l'on puisse modifier le $\mathrm{pH}$ du sol au voisinage des racines. Or, le Fomes annosus provoque une maladie qui est plus grave lorsque le $\mathrm{pH}$ du sol devient supérieur à 6. La modification du pH de la rhizosphère par l'intermédiaire des exsudats racinaires, ne serait donc probablement pas sans influence sur le développement de cette maladie. 